INVESTIGACIÓN

https://doi.org/10.15198/seeci.2019.49.19-37

Recibido: 19/08/2017 --- Aceptado: 22/02/2019--- Publicado: 15/07/2019

\title{
LA POBLACION SÉNIOR Y SU PERCEPCIÓN DE LA PUBLICIDAD: INFLUENCIA DEL MEDIO Y DEL EMISOR
}

\section{THE SENIOR POPULATION AND ITS PERCEPTION OF ADVERTISING: INFLUENCE OF THE MEDIA AND THE ISSUER}

\section{(8) Belén Ávila-Rodríguez-de-Mier': ESIC Business \& Marketing School.} España.

belen.avila@esic.edu

Noemí Martín-García: Universidad de Valladolid. España.

noemicarmen.martin@uva.es

\section{RESUMEN}

El acelerado envejecimiento de la población ha hecho que los mayores se estén convirtiendo en un público objetivo de alto interés para algunas marcas. Debido a ello, este trabajo se plantea con la finalidad de conocer la opinión que los sénior tienen de la publicidad y el grado de credibilidad que dan a los anuncios en función del emisor y del medio en el que sean expuestos. La metodología utilizada para dar respuesta a este objetivo está basada en entrevistas realizadas a individuos de 65 a 84 años con hábitos de envejecimiento activo y residentes en Segovia capital $(\mathrm{N}=184)$. Los resultados muestran cierto rechazo a la publicidad, especialmente entre los varones y los más jóvenes (65 a 74 años), a pesar de que se reconozca su utilidad. Además, la publicidad en radio y la publicidad institucional son percibidas como las más creíbles en contraposición con la emitida en internet y la proveniente de los partidos políticos. Su originalidad radica en ofrecer una información novedosa y segmentada que pueda ayudar a plantear estrategias de comunicación dirigidas a impactar a un público objetivo tan heterogéneo y desconocido como el sénior.

PALABRAS CLAVE: sénior - publicidad - credibilidad - medios publicitarios anunciantes - audiencia - público objetivo.

\section{ABSTRACT}

The accelerated aging of the population has caused the older ones to become a target group of high interest for some brands. Therefore, this work is proposed to know the

${ }^{1}$ Belén Ávila-Rodríguez-de-Mier. ESIC Business \& Marketing School. Departamento de Comunicación y Publicidad.

belen.avila@esic.edu 
Ávila-Rodríguez-de-Mier; B., y Martín-García, N. La población sénior y su percepción de la publicidad: influencia del medio y del emisor

opinion that seniors have about advertising and the degree of credibility they give to the ads depending on the issuer and the media that displays them. The methodology used to respond to this objective is based on interviews with individuals from 65 to 84 years old with active aging habits and living in Segovia capital $(N=184)$. The results show some reluctance to advertising, especially among men and the youngest (65 to 74 years old), even though its usefulness is recognized. In addition, radio advertising and institutional advertising are perceived as more credible as opposed to the one issued on the internet and that other coming from political parties. This work is the starting point of a line of research aimed at knowing the behavior of seniors in their role as consumers of advertising. Its originality lies in offering a new and segmented information that can help plan communication strategies aimed at impacting a target group as heterogeneous and unknown as the senior.

KEY WORDS: senior - advertising - credibility - advertising media - advertisers; audience - target group.

\section{A POPULAÇÃO SÊNIOR E SUA PERCEPÇÃO DA PUBLICIDADE: INFLUÊNCIA DO MEIO DE COMUNICAÇÃO E DO EMISSOR}

\section{RESUME}

O acelerado envelhecimento da população fez com que as pessoas idosas se convertam em um público objetivo de alto interesse para algumas marcas. Devido a isso, este trabalho se propõe com a finalidade de conhecer a opinião que os idosos têm da publicidade e o grau de credibilidade que dão aos anúncios em função do emissor e do meio de comunicação em que sejam expostos. A metodologia utilizada para dar resposta a este objetivo está baseada em entrevistas realizadas a indivíduos de 65 a 84 anos com hábitos de envelhecimento ativo e residentes em Segóvia capital $(\mathrm{N}=184)$. Os resultados mostram certo rechaço a publicidade, especialmente entre os homens e os mais jovens (65 a 74), apesar de que reconhecem sua utilidade. Ademais, a publicidade em rádio e a publicidade institucional são percebidas como as mais credíveis em contraposição com a emitida em internet e a proveniente dos partidos políticos. Sua originalidade radica em oferecer uma informação nova e segmentada que possa ajudar a levantar estratégias de comunicação dirigidas a impactar a um público objetivo tão heterogênico e desconhecido como são os idosos.

PALAVRAS CHAVE: idosos - publicidade - credibilidade - meios publicitários anunciantes - audiência - público objetivo.

\section{Cómo citar el artículo:}

Ávila-Rodríguez-de-Mier; B., y Martín-García, N. (2019). La población sénior y su percepción de la publicidad: influencia del medio y del emisor. [The senior population and its perception of advertising: influence of the media and the issuer]. Revista de Comunicación de la SEECI, 49, 19-37. doi: http://doi.org/10.15198/seeci.2019.49.19-37 Recuperado de http://www.seeci.net/revista/index.php/seeci/article/view/550 
Ávila-Rodríguez-de-Mier; B., y Martín-García, N. La población sénior y su percepción de la publicidad: influencia del medio y del emisor

\section{INTRODUCCIÓN}

El envejecimiento paulatino de la población y el creciente atractivo comercial del público sénior está haciendo que los individuos de 65 y más años estén adquiriendo gran relevancia para el campo publicitario. La ancianidad de la población española empezaba a manifestarse en el año 2000 cuando la proporción de personas mayores de 64 años superaba en un $3 \%$ a la de los menores de 16 años. Una tendencia que alcanzó un record histórico en el año 2017 con un índice de envejecimiento de 118 (Instituto Nacional de Estadística -INE-, s.f.), es decir, que por cada 100 niños menores de 16 años había 118 adultos de 65 años y más. A principios de 2017, el $19.9 \%$ de la población (8.7 millones) eran personas mayores de 64 años (INE, 2016b) y, de mantenerse la tendencia demográfica actual, se estima que este porcentaje podrá alcanzar el $25.6 \%$ ( 11,7 millones) en 2031 y el $34.6 \%$ (14.2 millones) en 2066 (INE, 2016a).

Este fenómeno de envejecimiento demográfico ha empezado a tratarse desde la economía del envejecimiento y la sociología de la vejez como una oportunidad de desarrollo e innovación (Bazo, 1992; Sánchez-Vera, 1996, 2000; Muñoz, Gallego y González, 2015). Y, en el sector del marketing y la publicidad, más que como un problema, se está empezando a gestionar como una oportunidad de negocio (Sánchez-Vera, 1996; Grande, 2001/2002; Escario, 2002; Furlong, 2007). De hecho, según un estudio realizado por Kantar Worldpanel en 2017, los hogares habitados por los más mayores concentran el $29 \%$ del gasto en los productos de gran consumo (alimentación, bebidas, droguería y perfumería), una cifra que se estima ascenderá al $40 \%$ en el 2030 . Todos estos datos sugieren que la supervivencia de muchas empresas pase por la reorientación a un mercado cada vez más envejecido (Wallace, 2000; Estrada, Sánchez, Moliner y Fandos, 2010) y del que, a día de hoy, aun se sabe poco sobre cómo percibe la publicidad. En este sentido apunta Mancebo-Aracil (2014) al afirmar que los principales estudios que relacionan a la población sénior con la publicidad y los medios de comunicación giran más en torno a la manera en que se trata y representa a este colectivo que a la opinión que este tiene de la publicidad. Ejemplo de ello es el estudio de Ramos-Soler y Carretón-Ballester (2012) acerca del uso que se hace de la imagen de los más mayores en los anuncios de televisión.

La importancia de la publicidad para los medios de comunicación queda reflejada en los más de cinco millones de euros invertidos en medios convencionales en el año 2017 y que representan el $0.46 \%$ del PIB (Infoadex, 2018). Este sector de los medios de comunicación se caracteriza por tener un modelo mixto de financiación en el que los ingresos procedentes de la publicidad ocupan un lugar destacado (Kind, Nilssen y Sørgard, 2005; Cea-Esteruelas, 2013; Campos-Freire, 2015). Entre los medios y las marcas anunciantes existe una fuerte relación de dependencia. Por un lado, los medios venden audiencias de alto atractivo comercial. $Y$, por otro lado, las marcas buscan una 'masa' específica de audiencia que contacte con su mensaje y lo recuerde en el momento justo de la decisión de compra. Este recuerdo del mensaje publicitario, además de por sus peculiaridades creativas, depende de factores tales como la credibilidad, el prestigio y la capacidad de influencia que tanto el medio

Revista de Comunicación de la SEECI. 15 julio, 2019 / 15 noviembre, 2019, no 49, 19-37 
Ávila-Rodríguez-de-Mier; B., y Martín-García, N. La población sénior y su percepción de la publicidad: influencia del medio y del emisor

como el anunciante ejercen sobre la audiencia (Victoria-Mas y Lacasa-Mas, 2015; Ávila-Rodríguez-de-Mier, 2016). De ahí, el creciente interés de los medios por crear y desarrollar marcas creíbles y con valor que les garanticen un compromiso o engagement con su audiencia (Arrese, 2013; Calvo-Porral, Martínez-Fernández y Juanatey-Boga, 2014). Y, además, les generen unos niveles de confiabilidad, notoriedad, prestigio social y consumo que repercutan directamente en sus ingresos (Choi, Watt y Lynch, 2006; Roses y Gómez-Campos, 2015).

Este estudio parte de la idea de que la credibilidad es el resultado de un proceso en el que las personas encuestadas evalúan, desde la experiencia emocional subjetiva, la fiabilidad de la fuente, del emisor, del contenido o de cualquier otro elemento informativo que pueda afectar al objeto de estudio (Hovland y Weiss, 1951; Metzger, Flanagin, Eyal, Lemus y Mccann, 2003). En este sentido apunta Del Barrio (2002) al manifestar que "si un determinado mensaje publicitario es percibido como muy poco creíble por parte de la audiencia, ello va a afectar negativamente a la aceptación del mensaje y, por ende, a la marca" (Del Barrio, 2002, p. 129). De ahí, la importancia de conocer la credibilidad que la audiencia sénior otorga al mensaje publicitario en función del medio en el que se expone y del tipo de anunciante que la emite.

Las investigaciones sobre la credibilidad en el entorno de los medios de comunicación son tratadas desde diferentes perspectivas convirtiéndose en relativamente habituales para el campo académico y el profesional. Prueba de ello son las aportaciones de autores como Rastrollo (2010) en su estudio sobre la credibilidad organizativa en la empresa informativa actual. La autora concluye en su investigación que la credibilidad es un indicador del buen hacer de la empresa que se convierte en un activo intangible de gran valor que trasciende a la calidad de la noticia. Por su parte, Roses y Farias-Batlle, (2012) afirman que la percepción de la credibilidad se encuentra muy asociada al nivel de estudios. Así, por ejemplo, aunque la televisión resulta ser el medio más creíble, este puesto pasa a ser ocupado por la prensa entre las personas con más formación. Desde otra perspectiva, Calvo-Porral et al. (2014) analizan la credibilidad de los diarios generalistas y confirman su efecto positivo en el comportamiento del consumidor y en la fidelidad y predisposición a aceptar nuevos productos y formatos. Por último, cabe resaltar el estudio realizado por la empresa Kantar en 2017 a 8.000 personas de diversos países como Brasil, Francia, Reino Unido y Estados Unidos. La multinacional determina que la confianza en los medios tradicionales frente a los digitales se ha visto reforzada a partir del fenómeno de las noticias falsas o fake news. Además, afirman que las revistas impresas de información general son para el $72 \%$ de los encuestados el medio que más confianza les genera mientras que las redes sociales son consideradas veraces por tan solo el 33\% de los encuestados. De acuerdo con las conclusiones de este estudio, la profundidad con la que se cubren las noticias es lo que mantiene a los medios tradicionales como más creíbles que los digitales (MarketingNews.es, 2017).

Sin embargo y como se apuntaba con anterioridad, las investigaciones sobre la credibilidad de la información publicitaria son menos numerosas. En este sentido, destacan las aportaciones de autores como Del Barrio (2002) o Zapata y Martínez- 
Ávila-Rodríguez-de-Mier; B., y Martín-García, N. La población sénior y su percepción de la publicidad: influencia del medio y del emisor

Caro (2016). Del Barrio (2002) demostró que la población percibe la publicidad comparativa como menos creíble que la no comparativa. Por su parte, Zapata y Martínez-Caro (2016) concluyeron que para el ámbito de los seguros, la honradez y experiencia del prescriptor son factores decisorios en la intención de compra mientras que su aspecto físico no lo es tanto.

Dentro del grupo de estudios que relacionan la credibilidad con la publicidad son prácticamente inexistentes las investigaciones que se enfocan en el público sénior. A este respecto, dentro del campo académico, encontramos la investigación de Estrada et al., (2010) sobre la actitud de las personas mayores ante los anuncios. El estudio concluye que, debido a las limitaciones que las personas mayores tienen para registrar, memorizar y recuperar la información, "precisan de una fuerte racionalización del mensaje, de una búsqueda pragmática entre sus conocimientos, experiencias y recuerdos, concediendo más importancia a sus pensamientos que al propio anuncio en si" (Estrada et al., 2010, p. 159). Dentro del campo profesional destaca el trabajo de ámbito internacional realizado por la empresa Nielsen en el año 2015 acerca de la confianza en la publicidad. La multinacional afirma que los anuncios de televisión, seguidos por los de prensa y revistas son los más confiables y los emitidos en el medio cine resultan ser los menos creíbles. A pesar de que el estudio permite segmentar por país, sexo y edad, la captación de la muestra se realiza teniendo únicamente en cuenta a los usuarios de internet por lo que consideramos que sus datos no son lo suficientemente representativos para el público sénior donde, según el INE (2017), únicamente un 38.0\% de los individuos de 65 a 74 años afirma utilizar internet una vez por semana.

Una vez analizado el conjunto de investigaciones tanto académicas como profesionales que vinculan la credibilidad de la publicidad con la población sénior, descubrimos ciertas carencias. Entre ellas, la no existencia de estudios en los que se asocie la percepción de la credibilidad publicitaria de la población sénior española con sus percepciones sobre el canal y el emisor de la publicidad. Por tanto, nos encontramos ante un campo bastante inexplorado sobre el que pretendemos obtener nuevos conocimientos que puedan llegar a tener implicaciones futuras tanto en el mundo académico como en el profesional.

\section{OBJETIVOS}

La investigación se plantea como un estudio exploratorio que tiene como objetivo general conocer la opinión que la población sénior (individuos de 65 a 84 años) tiene sobre la publicidad y al grado de credibilidad que le otorga en función del canal y del emisor. El estudio parte de la hipótesis de que, dentro del público sénior, el sexo y la edad determinan la percepción que se tiene de la publicidad. La confirmación de dicha hipótesis pasa por alcanzar los siguientes objetivos específicos:

1. Conocer la opinión del público sénior sobre la publicidad en lo que se refiere a sus gustos, su vinculación al coste del producto y su utilidad como información comercial. Estos datos nos permiten tener una primera aproximación de la visión que la población sénior (65 a 84 años) tiene de la publicidad. 
2. Averiguar el grado de credibilidad que otorga el público sénior a la publicidad en función del canal/medio en el que se emite y del emisor/tipo de anunciante que se publicita. Esta información influye en la credibilidad del anuncio y, por ende, a en la aceptación de los mensajes emitidos por las marcas.

\section{METODOLOGÍA}

Debido a que los individuos de 65 y más años representan un colectivo heterogéneo y disperso que no puede ser considerado un grupo social (Grande, 1993; Sánchez-Vera, 1996; Bódalo, 2003), la consecución de los dos objetivos específicos se plantea desde cuatro segmentos comparables dos a dos. Por un lado, se ha dividido a la población por edad teniendo en cuenta las directrices de Chackiel (2001) que agrupa a los sénior en Tercera edad (65 a 74) y Cuarta edad (75 a 84) atendiendo a que los primeros años están marcados por la jubilación mientras que los segundos se encuentran inmersos en una fase de mayor dependencia y deterioro. $\mathrm{Y}$, por otro lado, se han repartido en función del sexo, diferenciando entre hombres y mujeres.

En lo que a la metodología se refiere, se trata de una investigación básica, no experimental, seccional y exploratoria (Del Rio y Velázquez, 2005). Para su desarrollo se utilizó el método de la encuesta descriptiva basado en las directrices de Wimmer y Dominick (1996). El universo de estudio fue la población residente en Segovia capital con edades comprendidas entre los 65 y 84 años, conformada por un total de 9.321 individuos (Ayuntamiento de Segovia, 2015). El estudio considera los 84 años como la edad máxima del universo teniendo en cuenta los datos de la esperanza de vida de la población española que publica el Instituto Nacional de Estadística -80.4 años para los hombres y 85.9 años para las mujeres (INE, 2016b)-. Para el análisis se eligió una muestra de conveniencia compuesta por 184 individuos activos con una distribución de: $36 \%$ Hombres y $64 \%$ Mujeres; $57 \%$ Tercera edad (65 a 74 años) y $43 \%$ Cuarta edad (75 a 84 años).

Las encuestas se realizaron durante los meses de octubre y noviembre de 2017 y, como se buscaban personas en proceso de un envejecimiento activo, se realizaron en la Universidad de la Experiencia, escuela de la Universidad de Valladolid para mayores de 64 años y en los cursos de gimnasia y estimulación cognitiva dentro de las Aulas municipales para mayores de 64 años que organiza la Concejalía de Servicios Sociales, Igualdad, Sanidad y Consumo del Ayuntamiento de Segovia dentro del Programa de envejecimiento activo².

El cuestionario fue diseñado y administrado teniendo en cuenta las posibles deficiencias cognitivas y visuales de los entrevistados. Para ello, se tuvieron en cuenta aspectos tales como el tamaño de la letra (Arial 13), el espacio interlineado

\footnotetext{
${ }^{2}$ Se agradece la colaboración de Jesús Cruz (Profesor de Psicomotricidad para adultos), Jesús García (Técnico de Servicios Sociales del Ayuntamiento de Segovia), Sonia García (Profesora de Estimulación cognitiva), Juan Carlos Manrique (Coordinador de la Universidad de la Experiencia).
} 
$(1,5)$ o la entrega de bolígrafos de punta gruesa y fácil escritura. La herramienta fue testada previamente $y$, como consecuencia de los resultados de la prueba piloto, se sustituyó la escala intermedia de las respuestas -'Ni creíble, ni no creíble' en favor de 'No sabe/No contesta'- para evitar la confusión y/o el hastío en los entrevistados.

Con la finalidad de dar respuesta al primero de los objetivos específicos y, por tanto, conocer la opinión del público sénior sobre la publicidad en lo que se refiere a sus gustos, su vinculación al coste del producto y su utilidad como información comercial, se plantearon tres preguntas. En la primera de ellas, los encuestados fueron preguntados sobre si les gustaba la publicidad. Una vez resuelta, se les preguntó sobre si consideraban que esta encarecía el precio de los productos. Y por último, se les preguntó sobre si consideraban de utilidad la información suministrada por los anuncios. Las respuestas a estas preguntas eran cerradas y de elección única -Si, No, A veces, No sabe/No contesta-.

Para dar respuesta al segundo de los objetivos específicos y averiguar el grado de credibilidad que le otorga el público sénior a la publicidad según sea el medio en el que se emite y el emisor que se publicita, se realizaron dos preguntas. Primeramente, los encuestados fueron preguntados por el grado de credibilidad de la publicidad realizada en televisión, radio, prensa, revistas, internet y exterior. Y, seguidamente, por el grado de credibilidad de la publicidad realizada por los siguientes tipos de empresas/productos: Banca y Seguros (publicidad financiera); Laboratorios farmacéuticos; Estado, Comunidades y Ayuntamiento (publicidad institucional); Partidos políticos durante periodo electoral (publicidad política); Cosmética femenina y masculina; Gran consumo (alimentación, aseo y limpieza); Motor (coches y motos) y Grandes almacenes o superficies. Las respuestas a estas preguntas eran cerradas y de elección de escala -Muy creíble, Bastante creíble, Poco creíble, Nada creíble y No sabe/No contesta-.

El cuestionario se distribuyó a grupos de entre quince y treinta personas que los cumplimentaron bajo supervisión. El tiempo para realizarlo no superó en ninguno de los grupos los treinta minutos (Wimmer y Dominick, 1996). Con estas premisas, se consiguió una tasa de respuesta del $85 \%$.

\section{RESULTADOS}

Con objeto de presentar los resultados de manera clara, estos se han estructurado en cinco epígrafes que coinciden con las cinco preguntas realizadas en el cuestionario y que dan respuesta, de manera individual, a los dos objetivos específicos de la investigación y, de manera conjunta, a la hipótesis.

\subsection{Sobre el gusto por la publicidad}

La primera pregunta versó sobre el gusto por la publicidad y tenía por objetivo obtener una primera aproximación de la opinión que los sénior tienen sobre esta. Únicamente el $15.2 \%$ de los encuestados respondieron que 'si' les gustaba la 
Ávila-Rodríguez-de-Mier; B., y Martín-García, N. La población sénior y su percepción de la publicidad: influencia del medio y del emisor

publicidad frente al $38.0 \%$ que contestó que 'no' y el $40.8 \%$ que afirmaron que sólo les gustaba 'a veces'.

Por sexo, las mujeres se muestran bastante más atraídas por la publicidad que los hombres $(18.6 \%$ de las mujeres contestó que 'si' les gustaba la publicidad frente a un $9.0 \%$ de los hombres), siendo estos últimos los que mostraron un rechazo más tajante hacía la publicidad $(45.5 \%$ de respuestas negativas en hombres frente a un $33.9 \%$ en mujeres). Para comprobar si se trataba de diferencias estadísticamente significativas se utilizó el contraste Chi-cuadrado $\left(\mathrm{X}^{2}\right)$ que arrojó un valor de 5.67 con un grado de libertad (g.l) de 3 y un coeficiente de correlación de Pearson $(p)$ de .101, lo que demuestra que no existen diferencias notables en el gusto por la publicidad entre ambos sexos aunque si se observe una tendencia (Tabla 1).

Por grupos de edad, los individuos de 75 a 84 años se muestran más proclives a la publicidad que los 65 a 74 años $(21.3 \%$ de la Cuarta edad contestó que 'si' le gustaba la publicidad frente al $10.6 \%$ de la Tercera edad). El estadístico de contraste sigue mostrando que no existen diferencias significativas entre ambos grupos de edad aunque si se observe cierta tendencia $\left(X^{2}=7.59 ; 3\right.$ g.l.; $\left.p=.055\right)$ (Tabla 1$)$.

Tabla 1. El gusto por la publicidad de los sénior.

\begin{tabular}{|cccccc|}
\hline \multicolumn{7}{|l|}{ Pregunta 1: cLe gusta la publicidad? } \\
\hline \multicolumn{7}{|c|}{ Hombres } & Mujeres & 65 a 74 años & 75 a 84 años & Total \\
\hline $\mathrm{Si}$ & $9.1 \%$ & $18.6 \%$ & $10.6 \%$ & $21.3 \%$ & $15.2 \%$ \\
No & $45.5 \%$ & $33.9 \%$ & $44.2 \%$ & $30.0 \%$ & $38.0 \%$ \\
A veces & $42.4 \%$ & $39.8 \%$ & $37.5 \%$ & $45.0 \%$ & $40.8 \%$ \\
NS/NC & $3.0 \%$ & $7.6 \%$ & $7.7 \%$ & $3.8 \%$ & $6.0 \%$ \\
\hline$X^{2}$ & $5.67 ; 3$ g.l. $(p=.101)$ & $7.59 ; 3$ g.l. $(p=.055)$ \\
\hline
\end{tabular}

Fuente: Elaboración propia.

\subsection{Sobre la creencia de que la publicidad encarece los productos}

La segunda pregunta realizada con el objetivo de conocer la opinión general de los sénior acerca de la publicidad estaba dirigida a descubrir si este segmento consideraba que los anuncios encarecen el precio final del producto. Esta afirmación fue compartida por el $67.4 \%$ de la muestra mientras que un $10.9 \%$ de los encuestados opinó que la publicidad no influye en el precio y un $14.1 \%$ que los encarece 'a veces'. En esta ocasión, la variable sexo no resultó discriminante ya que contestaron afirmativamente el $66.7 \%$ de los hombres y el $67.8 \%$ de las mujeres. El estadístico de contraste muestra que no existen diferencias significativas entre ambos sexos $\left(X^{2}=.56 ; 3\right.$ g.l.; $\left.p=.906\right)$.

Aunque la edad tampoco se presenta como un factor discriminante $\left(X^{2}=1.36 ; 3\right.$ g.l.; $\mathrm{p}=.71$ ), la Cuarta edad, con un $78.8 \%$ de 'si + a veces', se mostró algo más 
Ávila-Rodríguez-de-Mier; B., y Martín-García, N. La población sénior y su percepción de la publicidad: influencia del medio y del emisor

tolerante con los efectos de la publicidad sobre el precio de los productos que la Tercera con un $83.7 \%$ de 'si + a veces' (Tabla 2 ).

Tabla 2. La creencia de que la publicidad encarece los productos.

\begin{tabular}{|c|c|c|c|c|c|}
\hline \multicolumn{6}{|c|}{ Pregunta 2: ¿Considera que la publicidad encarece el precio de los productos? } \\
\hline & Hombres & Mujeres & 65 a 74 años & 75 a 84 años & Total \\
\hline $\mathrm{Si}$ & $66.7 \%$ & $67.8 \%$ & $68.3 \%$ & $66.3 \%$ & $67.4 \%$ \\
\hline No & $12.1 \%$ & $10.2 \%$ & $8.7 \%$ & $13.8 \%$ & $10.9 \%$ \\
\hline A veces & $15.2 \%$ & $13.6 \%$ & $15.4 \%$ & $12.5 \%$ & $14.1 \%$ \\
\hline NS/NC & $6.1 \%$ & $8.5 \%$ & $7.7 \%$ & $7.5 \%$ & $7.6 \%$ \\
\hline$X^{2}$ & \multicolumn{2}{|c|}{$.56 ; 3$ g.l. $(p=.906)$} & \multicolumn{2}{|c|}{$1.36 ; 3$ g.l. $(p=.71)$} & \\
\hline
\end{tabular}

Fuente: Elaboración propia.

\subsection{La utilidad de la información publicitaria}

La tercera de las preguntas que permitió formar esa primera aproximación a la opinión que la población sénior tiene sobre la publicidad se basó en descubrir si consideraban de utilidad la información que les otorga esta práctica comercial. En este caso, el $26.1 \%$ de los encuestados manifestó que los mensajes publicitarios 'si' facilitan una información de utilidad frente a un $43.5 \%$ que consideró que este beneficio sólo se produce 'a veces'. Únicamente el $19.0 \%$ de la muestra fue rotunda en afirmar que estos mensajes 'no' son útiles.

Por sexo, las mujeres se mostraron bastante más convencidas de la utilidad de la información publicitaria que los hombres (32.2\% vs. $15.2 \%)$, mucho más reacios a atribuir algún provecho informativo a los anuncios. En este caso, el estadístico de contraste indicó que no existen diferencias significativas entre ambos sexos aunque si se observa una tendencia $\left(X^{2}=7,19 ; 3\right.$ g.l.; $\left.p=.066\right)$.

La variable edad muestra como la Cuarta edad otorga una mayor utilidad a la información publicitaria que la Tercera edad (20.2\% Tercera vs. 33.8\% Cuarta). A pesar de ello, la Tercera edad afirma en un porcentaje mayor (50.0\%) que esta utilidad se produce 'a veces' frente al $35.0 \%$ de la Cuarta edad que opina de igual manera (Tabla 3). Por este motivo, el estadístico de contraste sigue manifestando que las diferencias entre los dos tramos de edad no son significativas $\left(X^{2}=5.35 ; 3\right.$ g.l.; $p=.148)$.

Tabla 3. La utilidad de la información publicitaria para los sénior.

\begin{tabular}{|c|c|c|c|c|c|}
\hline \multicolumn{6}{|c|}{$\begin{array}{l}\text { Pregunta 3: ¿Considera que la publicidad da al consumidor una información de } \\
\text { utilidad? }\end{array}$} \\
\hline & Hombres & Mujeres & 65 a 74 años & 75 a 84 años & Total \\
\hline $\mathrm{Si}$ & $15.2 \%$ & $32.2 \%$ & $20.2 \%$ & $33.8 \%$ & $26.1 \%$ \\
\hline No & $24.2 \%$ & $16.1 \%$ & $19.2 \%$ & $18.8 \%$ & $19.0 \%$ \\
\hline$A$ veces & $45.5 \%$ & $42.4 \%$ & $50.0 \%$ & $35.0 \%$ & $43.5 \%$ \\
\hline $\mathrm{NS} / \mathrm{NC}$ & $15.2 \%$ & $9.3 \%$ & $10.6 \%$ & $12.5 \%$ & $11.4 \%$ \\
\hline
\end{tabular}


Ávila-Rodríguez-de-Mier; B., y Martín-García, N. La población sénior y su percepción de la publicidad: influencia del medio y del emisor

\begin{tabular}{lll}
\hline$X^{2}$ & $7.19 ; 3$ g.l. $(p=.066)$ & $5.35 ; 3$ g.l. $(p=.148)$ \\
\hline
\end{tabular}

Fuente: Elaboración propia.

Una vez expuestos los resultados a las preguntas dirigidas a tener una primera aproximación de la visión que la población sénior tiene de la publicidad, se procede con los resultados de las preguntas realizadas para dar respuesta al segundo de los objetivos específicos consistente en averiguar el grado de credibilidad que el público sénior otorga a la publicidad en función del medio en el que se emite y del tipo de anunciante que se publicita. Los resultados se presentan en base a la diferencia entre las escalas negativas y las positivas ${ }^{3}$. Los porcentajes de respuesta de NS/NC (no sabe/no contesta) no son contemplados con objeto de no desvirtuar los resultados. Finalmente señalar que, para poder realizar la comparación de los grados de credibilidad entre sexos y edades, se calculó el índice estadístico de la afinidad de cada segmento en relación a la media de los dos segmentos analizados ${ }^{4}$.

\subsection{Sobre la credibilidad de la publicidad en función del medio}

La primera pregunta sobre la percepción de la credibilidad del público sénior se enfocó en la figura de los medios de comunicación -televisión, radio, prensa, revisas, exterior e internet- como canales a través de los que se transmite el mensaje publicitario. Los datos porcentuales muestran como en ninguno de los medios analizados la credibilidad positiva supera a la negativa. A pesar de ello, la radio y la prensa son los medios que presentan un mayor porcentaje de credibilidad positiva ( $38.6 \%$ y $27.6 \%$ respectivamente), mientras que internet y exterior fueron los medios considerados menos creíbles por los sénior con unos porcentajes de $84.6 \%$ y $81.9 \%$ de credibilidad negativa respectivamente (Tabla 4 ). El estadístico de contraste Chi-cuadrado refleja estas diferencias significativas en relación con el grado de credibilidad que otorgan los sénior a la publicidad emitida en los diferentes medios $\left(X^{2}=28,621 ; 5\right.$ g.l.; $\left.p<.000\right)$.

Por sexo, la publicidad emitida en radio resulto ser la más creíble tanto para las mujeres (43.9\%) como para los hombres (30.0\%). La publicidad en prensa diaria ocupó el segundo lugar en ambos casos (31.5\% mujeres y $21.4 \%$ hombres). En lo que a la publicidad televisiva se refiere, las mujeres también se mostraron más crédulas que los hombres (27.5\% mujeres vs. $17.2 \%$ hombres).

Los medios percibidos como menos creíbles por las féminas fueron las revistas e internet con tan sólo un $20 \%$ de credibilidad positiva cada uno $y$, en el caso de los hombres, internet $(8.3 \%)$ y exterior (11.5\%) (Tabla 4$)$. Los índices estadísticos de afinidad mostrados en la Tabla 5 , corroboran que las mujeres otorgan valores más altos de credibilidad a la publicidad que los hombres destacando los casos de internet

${ }^{3}$ Diferencia entre las escalas negativas de 'nada creíble' y 'poco creíble' frente a las escalas positivas de 'bastante creíble' y 'muy creíble'.

${ }^{4}$ Se agradece a la Dra. Belinda de Frutos Torres las ideas en torno a la explicación de los resultados.

Revista de Comunicación de la SEECI. 15 julio, 2019 / 15 noviembre, 2019, no 49, 19-37 
Ávila-Rodríguez-de-Mier; B., y Martín-García, N. La población sénior y su percepción de la publicidad: influencia del medio y del emisor

(130) y exterior (122). A pesar de ello, los estadísticos de contraste no muestran que existan diferencias significativas entre hombres y mujeres en relación a la percepción de la credibilidad de la publicidad emitida en los diferentes medios.

Por tramos de edad, la publicidad en radio fue la que obtuvo el mayor porcentaje de credibilidad positiva (36.0\% Tercera Edad vs. 37.3\% Cuarta edad), seguida de la publicidad en prensa (30.5\% Tercera vs. $23.8 \%$ Cuarta) y de la emitida en televisión (25.0\% Tercera vs. $22.1 \%$ Cuarta). Por el contrario, la publicidad que obtuvo unos valores más bajos de credibilidad en la Tercera edad fueron las revistas $(20.3 \%)$ e internet $(20.8 \%)$ y, en la Cuarta fueron internet $(6.5 \%)$ y exterior $(10.7 \%)$ (Tabla 4$)$. Observando los índices de afinidad recogidos en la Tabla 5, se puede ver que los individuos de la Tercera edad se mostraron más crédulos con la publicidad emitida en todos los medios que los de la Cuarta edad, un hecho que cobró especial relevancia en internet (135) y exterior (128). A pesar de ello, el estadístico de contraste Chi-cuadrado muestra que solo existen diferencias significativas en la variable de edad en el medio internet $\left(X^{2}=4.48 ; 1 \mathrm{~g} . \mathrm{l} ; \mathrm{p}=.034\right)$.

Tabla 4. Porcentaje de credibilidad de la publicidad en función del medio.

\begin{tabular}{|llllllll|}
\hline \multicolumn{2}{|l|}{$\begin{array}{l}\text { Pregunta 4: ¿Qué grado de credibilidad tiene para Ud. la publicidad realizada en los } \\
\text { siguientes medios? }\end{array}$} \\
\hline \multirow{2}{*}{ Credibilidad } & Radio & Prensa & Televisión & Revistas & Exterior & Internet \\
\hline \multirow{2}{*}{ Total } & Positiva & $38.6 \%$ & $27.6 \%$ & $23.8 \%$ & $19.1 \%$ & $18.1 \%$ & $15.4 \%$ \\
& Negativa & $61.4 \%$ & $72.4 \%$ & $76.3 \%$ & $80.9 \%$ & $81.9 \%$ & $84.6 \%$ \\
\hline \multirow{2}{*}{ Hombres } & Positiva & $30.0 \%$ & $21.4 \%$ & $17.2 \%$ & $17.9 \%$ & $11.5 \%$ & $8.3 \%$ \\
& Negativa & $70.0 \%$ & $78.6 \%$ & $82.8 \%$ & $82.1 \%$ & $88.5 \%$ & $91.7 \%$ \\
\hline \multirow{2}{*}{ Mujeres } & Positiva & $43.9 \%$ & $31.5 \%$ & $27.5 \%$ & $20.0 \%$ & $22.1 \%$ & $20.0 \%$ \\
& Negativa & $56.1 \%$ & $68.5 \%$ & $72.5 \%$ & $80.0 \%$ & $77.9 \%$ & $80.0 \%$ \\
\hline \multirow{2}{*}{65 a 74} & Positiva & $39.6 \%$ & $30.5 \%$ & $25.0 \%$ & $20.3 \%$ & $23.2 \%$ & $20.8 \%$ \\
& Negativa & $60.4 \%$ & $69.5 \%$ & $75.0 \%$ & $79.7 \%$ & $76.8 \%$ & $79.2 \%$ \\
\hline \multirow{2}{*}{75 a 84} & Positiva & $37.3 \%$ & $23.8 \%$ & $22.1 \%$ & $17.7 \%$ & $10.7 \%$ & $6.5 \%$ \\
& Negativa & $62.7 \%$ & $76.2 \%$ & $77.9 \%$ & $82.3 \%$ & $89.3 \%$ & $93.5 \%$ \\
\hline
\end{tabular}

Fuente: Elaboración propia.

Tabla 5. Índice de credibilidad publicitaria en función del medio.

\begin{tabular}{|cccccc|}
\hline $\begin{array}{l}\text { Pregunta 4: ¿Qué grado de credibilidad tiene para Ud. la publicidad realizada en los } \\
\text { siguientes medios? }\end{array}$ & \multicolumn{5}{c|}{} \\
\hline Medio & Hombres & Mujeres & 65 a 74 & 75 a 84 & Total \\
\hline Radio & 78 & 114 & 103 & 93 & 100 \\
Prensa & 78 & 114 & 111 & 97 & 100 \\
Televisión & 73 & 116 & 105 & 86 & 100 \\
Revistas & 93 & 104 & 106 & 93 & 100 \\
Internet & 54 & 130 & 135 & 42 & 100 \\
Exterior & 64 & 122 & 128 & 59 & 100 \\
\hline
\end{tabular}

Fuente: Elaboración propia.

Revista de Comunicación de la SEECI. 15 julio, 2019 / 15 noviembre, 2019, no 49, 19-37 
Ávila-Rodríguez-de-Mier; B., y Martín-García, N. La población sénior y su percepción de la publicidad: influencia del medio y del emisor

\subsection{Sobre la credibilidad de la publicidad en función del emisor}

La segunda pregunta sobre la percepción de la credibilidad publicitaria se centró en conocer la veracidad que el colectivo sénior otorgaba a los anuncios en función del tipo de anunciante que realiza la comunicación. A este respecto, el estudio destacó que ninguno de los emisores analizados presentaba valores de credibilidad positiva por encima de los valores de credibilidad negativa. A pesar de ello, el estadístico de contraste Chi-cuadrado mostró que existen diferencias significativas en la credibilidad dada por los sénior a la publicidad según el tipo de anunciante $\left(X^{2}=103.085 ; 7\right.$ g.l.; $p$ $\mathrm{p}<.000$ ). Los emisores publicitarios más veraces para la población sénior son la publicidad institucional (40.3\%) y la publicidad farmacéutica (29.5\%). Por el contrario, los porcentajes más bajos de credibilidad se otorgaron a la publicidad de productos cosméticos (6.0\%) y a la publicidad política (3.9\%) (Tabla 6).

Por sexo, las mujeres conceden mayor credibilidad positiva que los hombres a todos los emisores excepto a la publicidad institucional (33.7\% mujeres vs. $50.0 \%$ hombres) y a la emitida por los partidos políticos en periodo electoral ( $2.1 \%$ mujeres vs. $6.9 \%$ hombres). El estadístico de contraste Chi-cuadrado muestra que únicamente existe una diferencia estadística significativa entre ambos sexos en la publicidad de cosmética $\left(X^{2}=8.889 ; 1\right.$ g.l.; $\left.p=.003\right)$, aunque se observe una tendencia en la publicidad institucional $\left(X^{2}=3.955 ; 1\right.$ g.l.; $\left.p=.047\right)$ y en la de alimentos ( $X^{2}=4.245 ; 1 \mathrm{~g} . \mathrm{l}$; $\left.p=.039\right)$. Los valores de afinidad recogidos en la Tabla 7 ayudan a visualizar que la publicidad institucional y la de partidos políticos son las más creíbles para los hombres mientras que el resto de los emisores es son más confiables para las mujeres, sobre todo en el caso de la publicidad de gran consumo (132) y de productos cosméticos (130).

Por tramos de edad los datos muestran como a medida que avanza la edad de los encuestados disminuye el porcentaje de credibilidad positiva otorgada a los diferentes emisores, sobre todo en los anuncios institucionales (45.8\% Tercera edad vs. $25.5 \%$ Cuarta edad), los financieros (21.6\% Tercera vs. $12.5 \%$ Cuarta) y en los de motor (13.8\% Tercera vs. $7.9 \%$ Cuarta). Por el contrario, aumenta la credibilidad positiva en los anuncios de cosmética (5.6\% Tercera vs. $8.3 \%$ Cuarta) (Tabla 6). Los individuos de la Tercera edad se muestran más crédulos con la publicidad de los tipos de emisores analizados que los de la Cuarta edad a excepción de la publicidad de laboratorios farmacéuticos (30.1\% Tercera vs. 31.9\% Cuarta) y la de cosmética (5.6\% Tercera vs. 8.3\% Cuarta). A pesar de ello, el estadístico de contraste Chicuadrado solo muestra diferencias significativas entre los dos tramos de edad en la publicidad institucional $\left(X^{2}=8.463 ; 1\right.$ g.l.; $\left.\mathrm{p}=.004\right)$. Por su parte, el valor de la afinidad de la credibilidad sitúa como más veraces para la Tercera edad todos los emisores a excepción de la publicidad de laboratorios farmacéuticos (102 Tercera vs. 108 Cuarta) y de cosmética (93 Tercera vs. 139 Cuarta) que son más confiables para los individuos de la Cuarta edad (Tabla 7).

Revista de Comunicación de la SEECI. 15 julio, 2019 / 15 noviembre, 2019, n 49, 19-37 
Ávila-Rodríguez-de-Mier; B., y Martín-García, N. La población sénior y su percepción de la publicidad: influencia del medio y del emisor

Tabla 6. Porcentaje de credibilidad de la publicidad según en función del emisor.

\begin{tabular}{|c|c|c|c|c|c|c|c|c|c|}
\hline \multicolumn{10}{|c|}{$\begin{array}{l}\text { Pregunta 5: ¿Qué grado de credibilidad tiene para Ud. la publicidad realizada por los } \\
\text { siguientes tipos de empresas/productos? }\end{array}$} \\
\hline & 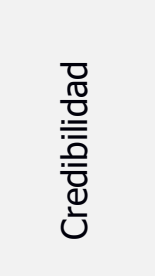 & 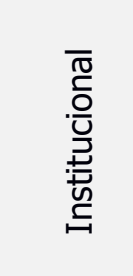 & 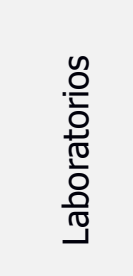 & 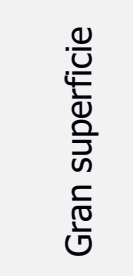 & 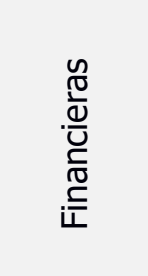 & 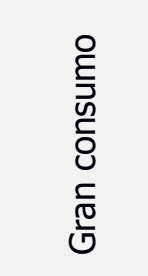 & $\begin{array}{l}\text { to } \\
\text { ¿o }\end{array}$ & 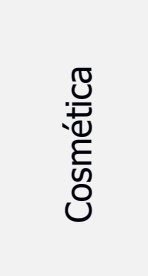 & 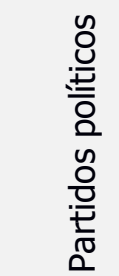 \\
\hline \multirow{2}{*}{$\begin{array}{l}\text { 㔄 } \\
\end{array}$} & Positiva & $40.3 \%$ & $29.5 \%$ & $20.8 \%$ & $17.8 \%$ & $14.9 \%$ & $11.4 \%$ & $6.0 \%$ & $3.9 \%$ \\
\hline & Negativa & $59.7 \%$ & $70.5 \%$ & $79.3 \%$ & $82.2 \%$ & $85.1 \%$ & $88.6 \%$ & $94.0 \%$ & $96.1 \%$ \\
\hline \multirow{2}{*}{ 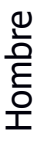 } & Positiva & $50.0 \%$ & $24.1 \%$ & $17.2 \%$ & $16.7 \%$ & $7.1 \%$ & $11.1 \%$ & $3.3 \%$ & $6.9 \%$ \\
\hline & Negativa & $50.0 \%$ & $75.9 \%$ & $82.8 \%$ & $83.3 \%$ & $92.9 \%$ & $88.9 \%$ & $96.7 \%$ & $93.1 \%$ \\
\hline \multirow{2}{*}{ 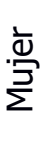 } & Positiva & $33.7 \%$ & $33.0 \%$ & $23.1 \%$ & $18.5 \%$ & $19.6 \%$ & $11.6 \%$ & $7.8 \%$ & $2.1 \%$ \\
\hline & Negativa & $66.3 \%$ & $67.0 \%$ & $76.9 \%$ & $81.5 \%$ & $80.4 \%$ & $88.4 \%$ & $92.2 \%$ & $97.9 \%$ \\
\hline \multirow{2}{*}{\begin{tabular}{l}
\multirow{N}{*}{} \\
$\tilde{0}$ \\
\end{tabular}} & Positiva & $45.8 \%$ & $30.1 \%$ & $22.5 \%$ & $21.6 \%$ & $16.9 \%$ & $13.8 \%$ & $5.6 \%$ & $4.1 \%$ \\
\hline & Negativa & $54.2 \%$ & $69.9 \%$ & $77.5 \%$ & $78.4 \%$ & $83.1 \%$ & $86.2 \%$ & $94.4 \%$ & $95.9 \%$ \\
\hline \multirow{2}{*}{$\begin{array}{l}\Phi \\
\infty \\
\tilde{N} \\
\end{array}$} & Positiva & $25.5 \%$ & $31.9 \%$ & $20.4 \%$ & $12.5 \%$ & $16.3 \%$ & $7.9 \%$ & $8.3 \%$ & $2.0 \%$ \\
\hline & Negativa & $74.5 \%$ & $68.1 \%$ & $79.6 \%$ & $87.5 \%$ & $83.7 \%$ & $92.1 \%$ & $91.7 \%$ & $98.0 \%$ \\
\hline
\end{tabular}

Fuente: Elaboración propia.

Tabla 7. Índice de credibilidad publicitaria en función del emisor.

Pregunta 5: ¿Qué grado de credibilidad tiene para Ud. la publicidad realizada por los siguientes tipos de empresas/productos?

\begin{tabular}{|cccccc|}
\hline Tipo de emisor & Hombre & Mujer & 65 a 74 & 75 a 84 & Total \\
\hline Institucional & 124 & 84 & 114 & 63 & 100 \\
Laboratorio farmacéutico & 82 & 112 & 102 & 108 & 100 \\
Entidad financiera & 94 & 104 & 122 & 70 & 100 \\
Grandes superficies & 83 & 111 & 108 & 98 & 100 \\
Gran consumo & 48 & 132 & 114 & 11 & 100 \\
Motor & 98 & 111 & 121 & 69 & 100 \\
Cosmética & 56 & 130 & 93 & 139 & 100 \\
Partidos políticos & 175 & 54 & 103 & 52 & 100 \\
\hline
\end{tabular}

Fuente: Elaboración propia. 
Ávila-Rodríguez-de-Mier; B., y Martín-García, N. La población sénior y su percepción de la publicidad: influencia del medio y del emisor

\section{CONCLUSIONES Y DISCUSIÓN}

Las respuestas a las diferentes cuestiones planteadas en la investigación permiten conocer la opinión que tiene el colectivo sénior acerca de la publicidad así como el grado de credibilidad que otorgan a los anuncios en función del medio en el que se exponen y del tipo de anunciante que los emite. Los resultados en conjunto muestran cierto rechazo a la publicidad por parte del colectivo sénior aunque también consideran que ofrece una información de utilidad. Así como, una baja incidencia de las variables sexo y edad en la percepción que dicho colectivo tiene de la publicidad, el liderazgo de la publicidad radiofónica en el ranking de credibilidad y la desconfianza generada por la publicidad emitida por los partidos políticos y las marcas de cosmética.

Como se indicaba anteriormente, la población sénior presenta cierto rechazo a la publicidad, una afirmación que se sustenta en el hecho de que, al 38.0\% de los entrevistados no les gusta la publicidad y al $40.8 \%$ sólo les gusta en ocasiones. Estos datos invitan a investigadores, marcas y agencias a profundizar sobre las necesidades y expectativas de los más mayores con el propósito de crear comunicaciones comerciales más atractivas para ellos. No obstante, los hombres se muestran más críticos con la publicidad que las mujeres (45.5\% vs. 33.9\%) y la Tercera edad más que la Cuarta edad (44.2\% vs. 30.0\%). Este hecho hace que conseguir un impacto publicitario efectivo resulte más complejo en estos dos subgrupos. Además, el $67.4 \%$ de los encuestados opina que la publicidad encarece el precio final de los productos, una idea que puede estar trabajando en contra de su buena imagen. A pesar de las anteriores afirmaciones - no les gusta y encarece el precio del producto- para el $69.9 \%$ de los encuestados la publicidad ofrece una información de utilidad, una circunstancia garantiza a las marcas la aceptación de sus mensajes publicitarios.

En lo relacionado con el grado de credibilidad que el público sénior otorga a los anuncios según el canal de emisión, la publicidad de radio es percibida como la más veraz $(38.6 \%$ credibilidad positiva) seguida de la de prensa diaria (27.6\%). Sin embargo, en ninguno de estos medios se observan diferencias significativas por sexo y edad. Este hecho ayuda a confirmar que ambos medios son los más valorados por la población sénior y, por tanto los más susceptibles de ofrecer a las marcas la posibilidad de generar engagement o compromiso con el público sénior. La publicidad en televisión, el medio con mayor penetración entre los más mayores, según el EGM, se erige en el tercer puesto con un $23.8 \%$ de credibilidad positiva. En contraposición, la publicidad en internet (15.4\%) es la que presenta un menor nivel de credibilidad, circunstancia en la que influye la brecha digital existente entre la población sénior y que justifica que la aceptación de la publicidad digital sea sensiblemente diferente entre la Tercera edad $(20.8 \%)$ y la Cuarta edad (6.5\%). De lo anterior se deduce que la inclusión de internet en campañas dirigidas al público sénior requiera de una cuidada selección de soportes y un tratamiento especial de la idea creativa. En relación al grado de credibilidad que otorgan los mayores a la publicidad en función de la fuente emisora, la investigación pone de relieve que la publicidad institucional y la farmacéutica son las que resultan más creíbles mientras 
Ávila-Rodríguez-de-Mier; B., y Martín-García, N. La población sénior y su percepción de la publicidad: influencia del medio y del emisor

que la publicidad de los partidos políticos y la de productos cosméticos son consideradas las menos fiables.

En conjunto, las diferentes respuestas aportadas por la muestra junto con los contrastes estadísticos Chi-cuadrado, invitan a descartar la hipótesis de que, dentro del público sénior, el sexo y la edad sean claramente determinantes en la percepción que se tiene de la publicidad. A pesar de ello, cabe destacar que, porcentualmente, a las mujeres les gusta más la publicidad y valoran más su utilidad, dos aspectos que ayudan a que se muestren más crédulas ante ella que los hombres. En cuanto al tipo de emisor, a pesar de que no confían realmente en ninguno, las mujeres otorgan más credibilidad a la publicidad institucional (33.7\%) y a la farmacéutica $(33.0 \%)$ y, entre los hombres, destaca el valor otorgado a la publicidad institucional $(50.0 \%)$. Por tramos de edad, el estudio demuestra que los individuos de la Cuarta edad tienen un mayor gusto por la publicidad y la consideran de mayor utilidad que los de la Tercera edad. A pesar de ello, se muestran menos crédulos con la publicidad emitida en los diferentes medios y con todos los emisores a excepción de la publicidad farmacéutica y de cosmética.

Los datos y conclusiones aportadas en este estudio pretenden ofrecer una información de utilidad a la hora de plantear estrategias de comunicación comercial dirigidas a impactar a un público objetivo tan desconocido como el sénior en sus diferentes segmentaciones. El trabajo es el punto de partida de una línea de investigación dirigida a conocer con profundidad cómo se comportan los individuos de 65 a 84 años en su papel de consumidores de comunicación comercial y de medios de comunicación publicitaria.

\section{REFERENCIAS}

Arrese, A. (2013). El valor de la marca periodística, en Toro J.M. \& Bel, I. (Eds.), Libro blanco de la prensa diaria, 2013 (pp.365-377). Madrid: AEDE.

Ávila-Rodríguez-de-Mier, B. (2016). El fenómeno mediático de la prensa gratuita en el marco de la Sociedad de la Información. España: Nacimiento, auge, crisis y convergencia (2000-2013), (pp. 319-321 y 364-373). Madrid: ESIC.

Ayuntamiento de Segovia (2016). Padrón Municipal Segovia 2016.

Bazo-Royo, M. T. (1992). La nueva sociología de la vejez: de la teoría a los métodos, en Reis: Revista Española de Investigaciones Sociológicas, 60, 75-90. doi: $10.2307 / 40183681$

Bódalo-Lozano, E. (2003). Aproximación sociológica a las necesidades y al consumo de los mayores, en Reis: Revista Española de Investigaciones Sociológicas, 103, 83-111. doi: $10.2307 / 40184554$

Calvo-Porral, C.; Martínez-Fernández, V. A, y Juanatey-Boga, O. (2014). Credibilidad de los medios de comunicación: análisis de la prensa diaria desde el 
Ávila-Rodríguez-de-Mier; B., y Martín-García, N. La población sénior y su percepción de la publicidad: influencia del medio y del emisor

comportamiento del consumidor, en El profesional de la información, 23(3), 300309. doi: 10.3145

Campos-Freire, F. (2015). Adaptación de los medios tradicionales a la innovación de los metamedios, en El profesional de la información, 24(4), 441-450. doi: 10.3145

Cea-Esteruelas, M.N. (2013). Economía de los cibermedios: modelos de ingresos y fuentes de financiación, en El profesional de la información, 22(4), 353-361. doi: 10.3145

Chackiel, J. (2001). El envejecimiento de la población latinoamericana, en Franco, R. (coord.), Sociología del desarrollo, políticas sociales y democracia (pp. 166-185). México: Siglo XXI Ediciones.

Choi, J.; Watt, J. H., y Lynch, M. (2006). Perceptions of News Credibility about the War in Iraq: Why War Opponents Perceived the Internet as the Most Credible Medium, en Journal of Computer-mediated Communication, 12(1), 209-229. doi: 10.1111

Del-Barrio-García, S. (2002): La credibilidad como elemento clave en la eficacia de la publicidad comparativa en prensa: un estudio experimental, en Investigaciones Europeas de Dirección y Economía de la Empresa, 8(1), 119-134. Recuperado de https://dialnet.unirioja.es/servlet/articulo?codigo $=251465$

Del-Rio, O., y Velázquez, T. (2005). Planificación de la investigación en Comunicación: fases del proceso, en Berganza-Conde, M.R \& Ruiz-San Román, J. A. (coord.), Investigar en Comunicación. Guía práctica de métodos y técnicas de investigación social en Comunicación (pp. 43-76). Madrid: Mc Graw Hill.

Escario, P. (2002). Marketing de la tercera edad, en IP Mark, 576, 37.

Estrada, M.; Sánchez, J., Moliner, M., y Fandos, J. C. (2010). Antecedentes y consecuencias de la actitud ante el anuncio en las personas mayores. Un análisis empírico, en Revista Innovar, 20(37), 149-162. Recuperado de http://www.redalyc.org/articulo.oa?id=81818989012

Furlong, M. S. (2007). Turning silver into gold: How to profit in the new boomer marketplace. Nueva Jersey (USA): Financial Times Prentice Hall.

Grande Esteban, I. (1993). Marketing estratégico para la tercera edad. Principios para atender a un segmento creciente. Madrid: ESIC.

Grande Esteban, I. (2001/2002). La tercera edad: un segmento emergente, en Comunicación y estudios universitarios, 11, 67-92. 
Ávila-Rodríguez-de-Mier; B., y Martín-García, N. La población sénior y su percepción de la publicidad: influencia del medio y del emisor

Hovland, C., y Weiss, W. (1951). The Influence of Source Credibility on Communication Effectiveness, en Public Opinion Quarterly, 15(4), 635-650. doi: 10.1086

INE (s.f.). Indicadores de Estructura de la Población. Índice de envejecimiento por provincia. Recuperado de http://www.ine.es/jaxiT3/Datos.htm?t=1489

INE (2016a). Proyecciones de Población 2016-2066. Nota de prensa 20-10-2016. Recuperado de http://www.ine.es/prensa/np994.pdf.

INE (2016b). Evolución de la esperanza de vida al nacimiento por periodo. Recuperado de

http://www.ine.es/jaxi/Datos.htm?path=/t00/mujeres hombres/tablas 2/10/\&fil=d 1g1.px

INE (2017). Encuesta sobre Equipamiento y Uso de Tecnologías de Información y Comunicación en los Hogares. Año 2017. Nota de prensa 05-10-2017. Recuperado de http://www.ine.es/prensa/tich_2017.pdf

Infoadex (2018). Resumen Estudio Infoadex de la inversión publicitaria en España en 2018. Madrid: Infoadex. Recuperado de http://www.infoadex.es/home/wpcontent/uploads/2018/02/Estudio-InfoAdex-2018.pdf

Kantar Worldpanel (2017). Seniors, la generación futura y presente. Recuperado de https://www.kantarworldpanel.com/es/Noticias/Seniors-la-generacion-futura-ypresente

Kind, H. J.; Nilssen, T., y Sørgard, L. (2005). Financing of media firms: Does competition matter?, en Memorandum, Department of Economics, University of Oslo, 2005(01). Recuperado de

http://citeseerx.ist.psu.edu/viewdoc/download?doi=10.1.1.485.1759\&rep=rep1\&ty pe $=$ pdf

Mancebo-Aracil, J. F. (2014). Mayores, publicidad y medios de comunicación: Una revisión teórica, en Historia y Comunicación Social, 19, 573-588. doi: 10.5209

MarketingNews.es (2017, 6 de noviembre). Lo que la "Posverdad" Esconde: los medios tradicionales ganan en confianza. Recuperado de http://www.marketingnews.es/tendencias/noticia/1110237029005/posverdadesconde-medios-tradicionales-ganan-confianza.1.html

Metzger, M.; Flanagin, A. J.; Eyal, K.; Lemus, D. R., y Mccann, R. M. (2003). Credibility in the 21st century: Integrating perspectives on source, message, and media credibility in the contemporary media environment, en Communication Yearbook, 27, 293-335. doi: 10.1080 
Ávila-Rodríguez-de-Mier; B., y Martín-García, N. La población sénior y su percepción de la publicidad: influencia del medio y del emisor

Muñoz Gallego, P. A.; González Benito, O., y Garrido Morgado, A. (2015). Estudio sobre las características y hábitos de comportamiento del segmento "plateado". Cetro Virtual sobre el envejecimiento. Fundación General de la Universidad de Salamanca.

Nielsen (2015). La confianza global de la publicidad. Estrategias para la obtención de logros en un panorama de medios en evolución. The Nielsen Company.

Ramos-Soler, I., y Carretón-Ballester, C. (2012). Presencia y representación de las personas mayores en la publicidad televisiva: el caso español, en Revista Española de Geriatría y Gerontología, 47(2), 55-61. doi: 10.1016

Rastrollo Horrillo, M.A. (2010). Credibilidad de la empresa informativa. Una perspectiva organizativa en Actas II Congreso Internacional Latina de Comunicación Social, Universidad La Laguna. Recuperado de http://www.revistalatinacs.org/10SLCS/actas 2010/Rastrollo.pdf

Roses, S. y Farias-Batlle, P. (2012). Credibilidad de los medios: un análisis bivariado de las opiniones de los españoles, en Revista Mediterránea de Comunicación, 3(1), 79-104. doi: 10.14198

Roses, S. y Gómez-Calderón, B. (2015). Credibilidad de los medios en España: divergencias de percepción y caracterización de los escépticos, en El profesional de la información, 24(4), 432-439. doi: 10.3145

Sánchez Vera, P. (1996). Tercera y cuarta edad en España desde la perspectiva de los hogares, en Revista Española de Investigaciones Sociológicas, 73, 57-79. Recuperado de https://dialnet.unirioja.es/servlet/articulo?codigo=761462

Sánchez Vera, P. (2000). Sociología de la vejez versus economía de la vejez, en Revista de Sociología, 61, pp. 39-88. doi: 10.5565

Victoria-Mas, M., y Lacasa-Mas, I. (2015). Gestión del valor de marca en las empresas de prensa. El caso de La vanguardia, en El profesional de la información, 24(4), 405-412. doi: 10.3145

Wallace, P. (2000). El seísmo demográfico. Madrid: Siglo XXI de España Editores.

Wimmer, R. D. y Dominick, J. R. (1996). La investigación científica de los medios de comunicación. Barcelona: Bosch.

Zapata, S. y Martínez-Caro, L. (2016). La influencia de la credibilidad de las celebridades en la publicidad, en Anuario de Jóvenes Investigadores, 9, 240-243. Recuperado de http://repositorio.upct.es/bitstream/handle/10317/5933/icc.pdf?sequence=1 
Ávila-Rodríguez-de-Mier; B., y Martín-García, N. La población sénior y su percepción de la publicidad: influencia del medio y del emisor

\section{AUTORAS:}

\section{Belén Ávila-Rodríguez-de-Mier}

Doctora en Sociología por la Universidad Pontificia de Salamanca. Executive Máster en Dirección Comercial y Marketing (CESMA Business School) y Licenciada en Publicidad y Relaciones Públicas (UCM). En la actualidad es profesora de grado y posgrado del área de Comunicación y Publicidad. Durante su trayectoria profesional trabajó en agencias como Leo-Burnett y McCann además de ocupar puestos de responsabilidad en los departamentos de marketing de Vodafone y Ferrovial Inmobiliaria. Su línea de investigación gira en torno a los medios de comunicación, las audiencias y la publicidad en el marco de la Sociedad de la Información.

belen.avila@esic.edu

Orcid ID: http://orcid.org/0000-0001-8381-4461

Google Scholar: https://scholar.google.es/citations?user=02JdxjMAAAAJ\&hl=es

\section{Noemí Martín-García}

Doctora por la Universidad de Valladolid (UVa) y Licenciada en Publicidad y RR.PP por la Universidad Complutense de Madrid con Curso Superior en Medios impartido por la Asociación de Agencias de Medios. Actualmente es profesora asociada en el grado de Publicidad y RR.PP de la UVa. Durante su trayectoria profesional ha trabajado para diferentes clientes tanto públicos como privados en las agencias de medios: Havas Media y Mindshareworld. Su línea de investigación se centra en la relación de la publicidad con los medios de comunicación y ha sido publicada en revistas como Icono 14 y El Profesional de la información.

noemicarmen.martin@uva.es

Orcid ID: http://orcid.org/0000-0003-3478-5021

Google Scholar:

https://scholar.google.es/citations?view_op=list_works\&hl=es\&user=_EXfBVUAAAAJ 